\section{Myeloid dysfunction in ALS}

The neurodegenerative disease amyotrophic lateral sclerosis (ALS) is linked to genetic mutations in C9orf72. In Science, O'Rourke et al. characterize mice deficient in C9orf72. Myeloid cells and microglia have more abundant expression of $C 9$ orf 72 than that of other immune cells and neurons. Loss of C9orf72 leads to an accumulation of debris-laden CD11 $b^{+}$myeloid cells in lymphoid tissues rather than overt neuronal defects. Mutant macrophages display defective endolysosomal function and increased content of LAMP-1+ lysosomes, which leads to increased production of reactive oxygen species and proinflammatory cytokines. Increased proinflammatory gene expression is also observed in brain tissues from a cohort of subjects with C9-associated ALS. These findings suggest that loss-of-function mutations of C9orf72 can impair normal phagocytic processing by myeloid cells and contribute to a chronic proinflammatory scenario that might affect neuronal function; however, additional factors probably influence the human disease.

Science 351, 1324-1329 (2016)

\section{Good cholesterol}

$\mathrm{CD}^{+} \mathrm{T}$ cells serve on the front line in battling tumors. In Nature, $\mathrm{Xu}$ and colleagues show that modulation of membrane cholesterol content can enhance the anti-tumor response by $\mathrm{CD} 8^{+} \mathrm{T}$ cells. Inhibition or deletion of the gene encoding the cholesterol-esterification enzyme ACAT1 leads to enhanced effector function of $\mathrm{CD}^{+} \mathrm{T}$ cells, including increased production of cytokines and granzymes in vitro and anti-tumor responses in vivo. Acat 1 is upregulated after activation of the $\mathrm{T}$ cell antigen receptor (TCR) in $\mathrm{CD}^{+} \mathrm{T}$ cells but not in $\mathrm{CD}^{+} \mathrm{T}$ cells. This response is concomitant with a reduction in the plasma-membrane cholesterol content, suggestive of a negative feedback mechanism for TCR signaling in immunological synapses. This hypothesis is supported by the enhanced formation of TCR microclusters and activation of downstream signaling components observed in the ACAT1-deficient $\mathrm{CD}^{+} \mathrm{T}$ cells. The findings suggest that modulation of cholesterol metabolism might have therapeutic benefits for promoting anti-tumor responses in the clinic.

Nature (16 March 2016) doi:10.1038/nature17412

\section{Club cells to the rescue}

Respiratory viral infection can result in a temporary and nonspecific enhancement in resistance to subsequent respiratory infection. In the Proceedings of the National Academy of Sciences USA, Heaton and colleagues demonstrate that initial infection with influenza $A$ virus renders mice more resistant to heterologous infection with influenza $B$ virus. This resistance depends on club cells, a type of exocrine cell present in the respiratory epithelium. Club cells that survive infection show enhanced type I interferon signaling, and prior infection results in alterations in the transcription of genes encoding chemokines and proinflammatory cytokines in the lungs. Nonspecific resistance to infection with a second virus begins to diminish 6 weeks after the initial infection and is almost completely lost by 12 weeks, presumably due to natural turnover of the club cells. The precise mechanism used by club cells in this nonspecific resistance remains unclear.

Proc. Natl. Acad. Sci. USA (21 March 2016) doi:10.1073/

pnas. 1522376113

\section{Gender imbalance}

Both female mice and female humans have a well-known predisposition to autoimmune disease. In the Journal of Clinical Investigation, Berrih-Aknin and colleagues demonstrate that sex hormones have an important influence on thymic central tolerance. Medullary thymic epithelial cells from female humans or from female mice have lower expression of AIRE, a transcription factor involved in imposing central tolerance, than those of their male counterparts. The expression of tissue-restricted antigens controlled by AIRE is similarly lower in females. Estrogen negatively regulates AIRE expression, and the effects of this female sex hormone are in turn antagonized by testosterone. Loss of the main estrogen receptor in medullary thymic epithelial cells from female mice restores the expression of AIRE and tissue-restricted antigens. Conversely, castration of male mice diminishes AIRE expression, and knockdown of AIRE enhances susceptibility to autoimmune thyroiditis. Sex hormones therefore serve an important role in shaping self-tolerance in the thymus and influence predisposition to autoimmunity.

J. Clin. Invest. (21 March 2016) doi:10.1172/JCI81894

\section{Platelet bias with age}

Aged hematopoietic stem cells (HSCs) show increased expression of myeloid-associated genes at the expense of the expression of lymphoid genes and diminished engraftment after transplantation. In Nature Communications, Grover et al. use single-cell transcriptomics to compare individual HSCs from young and aged mice. Young HSCs have increased expression of genes encoding cell-cycle regulators, while old HSCs show increased expression of genes encoding regulators of the immediate-early response to growth factors and megakaryocyte-platelet lineage programming. Aged HSC populations have an increased frequency of $\mathrm{Vwf}^{+}$ platelet-primed HSCs and an increase in the expression frequency of platelet-specific genes in all HSCs, which corresponds to a greater bias for platelet generation and less lymphoid potential than that of young HSCs. Deletion of FOG-1, which along with GATA-1 is required for platelet-lineage differentiation, restores the lymphoid-reconstitution potential of aged HSCs.

Nat. Commun. (24 March 2016) doi:10.1038/ncomms11075

\section{Beyond systems analysis}

RNA sequencing can be used to monitor nascent transcripts to provide insight into gene transcription independently of mRNA stability. In Cell, Smale and colleagues use RNA sequencing of nascent transcripts, chromatin immunoprecipitation followed by deep sequencing (ChIP-seq) and binding-motif data sets to delineate unique features of the transcription regulation of individual genes. Beyond statistical enrichment, careful analysis of motif strength, nascent versus existing mRNA, ChIP-seq peak strength and chromatin availability at promoters of primary and secondary response genes induced substantially (over tenfold) in lipid A-stimulated bone marrow-derived macrophages identifies distinct and unique regulatory mechanisms at promoters regulated by the transcription factors NF- $\kappa \mathrm{B}$ and IRF3. As such, a motif-strength threshold is required for the binding of NF- $\mathrm{KB}$ to promoters in vivo and delayed induction of particular genes is due to a requirement for nucleosome remodeling. Such quantitative, genecentric analysis also reveals that although IRF3 and NF- $\kappa \mathrm{B}$ cooperatively bind hundreds of genes, they co-regulate a very restricted number of genes, indicative of a highly targeted and subtle regulatory input. Cell 165, 165-179 (2016) 